This article has been downloaded from IOPscience. Please scroll down to see the full text article. (http://iopscience.iop.org/0953-8984/19/24/246225)

More related content is available

Download details:

IP Address: 149.156.194.12

The article was downloaded on 04/03/2008 at 11:49

Please note that terms and conditions apply. 


\title{
Multiple magnetic phase transitions in $\mathrm{Tb}_{3} \mathrm{Cu}_{4} \mathrm{Si}_{4}$
}

\author{
Ł Gondek ${ }^{1,6}$, A Szytuła ${ }^{2}$, D Kaczorowski ${ }^{3}$, A Szewczyk ${ }^{4}$, M Gutowska ${ }^{4}$ \\ and P Piekarz \\ ${ }^{1}$ Faculty of Physics and Applied Computer Science, AGH University of Science and Technology, \\ Mickiewicza 30, 30-059 Kraków, Poland \\ 2 M Smoluchowski Institute of Physics, Jagiellonian University, Reymonta 4, 30-059 Kraków, \\ Poland \\ ${ }^{3}$ W Trzebiatowski Institute of Low Temperature and Structure Research, Polish Academy of \\ Sciences, PO Box 1410, 50-950 Wrocław, Poland \\ ${ }^{4}$ Institute of Physics, Polish Academy of Sciences, Lotników 32/46, 02-668 Warszawa, Poland \\ ${ }^{5}$ H Niewodniczańki Institute of Nuclear Physics, Polish Academy of Sciences, Radzikowskiego \\ 152, 31-342 Kraków, Poland \\ E-mail: lgondek@agh.edu.pl
}

Received 11 January 2007, in final form 13 April 2007

Published 30 May 2007

Online at stacks.iop.org/JPhysCM/19/246225

\begin{abstract}
A polycrystalline sample of $\mathrm{Tb}_{3} \mathrm{Cu}_{4} \mathrm{Si}_{4}$ was investigated by means of magnetometric, electrical resistivity, thermopower and heat capacity measurements. This paper also includes reanalyses of former neutron diffraction data. The compound crystallizes in $\mathrm{Gd}_{3} \mathrm{Cu}_{4} \mathrm{Ge}_{4}$-type orthorhombic structure (Immm space group), in which the Tb ions occupy two inequivalent sites $(2 \mathrm{~d}$ and $4 \mathrm{e})$. The bulk results indicate that these two magnetic sublattices order antiferromagnetically at different temperatures, in agreement with the neutron diffraction data.
\end{abstract}

\section{Introduction}

The series of $\mathrm{R}_{3} \mathrm{~T}_{4} \mathrm{X}_{4}$ intermetallics $(\mathrm{R}=$ rare earth, $\mathrm{T}=d$-electron transition metal, $\mathrm{X}=\mathrm{p}$ electron element) attract much attention due to their complex magnetic properties [1-3]. They crystallize in the orthorhombic $\mathrm{Gd}_{3} \mathrm{Cu}_{4} \mathrm{Ge}_{4}$-type crystal structure (space group No. 71) [4], in which the rare earth ions occupy two different crystallographic sites: $2 \mathrm{~d}$ and $4 \mathrm{e}$. Each of the two magnetic sublattices orders antiferromagnetically at low temperatures with different Néel points and different propagation vectors. In a few compounds some spin reorientations have been observed in the ordered state. The general magnetic behaviour of these phases cannot be understood within the classical RKKY model: (i) the de Gennes scaling of ordering temperatures is clearly not obeyed, and (ii) changes in the propagation vector at one sublattice do not affect the other one [1-3]. Instead, it seems that in $\mathrm{R}_{3} \mathrm{~T}_{4} \mathrm{X}_{4}$ two magnetic sublattices order independently due to some local exchange interactions.

6 Author to whom any correspondence should be addressed. 
In this paper we discuss the magnetic behaviour of the silicide $\mathrm{Tb}_{3} \mathrm{Cu}_{4} \mathrm{Si}_{4}$. Our former neutron diffraction studies revealed a very complex magnetic phase diagram for this compound [1]. In the light of the new results presented in this work, the magnetic structure of this compounds must be revisited. Now, it is clear that ordering temperatures of both sublattices differ significantly. The ordering temperature of the $2 \mathrm{~d}$ sublattice is close to $48 \mathrm{~K}$, while the $4 \mathrm{e}$ sublattice becomes disordered just above $7 \mathrm{~K}$ only. Thus, a question arises why the strength of the exchange interactions at the two magnetic sites is so different that they result in distinctly dissimilar critical temperatures.

\section{Experimental details}

A polycrystalline sample of $\mathrm{Tb}_{3} \mathrm{Cu}_{4} \mathrm{Si}_{4}$ was prepared by arc melting stoichiometric amounts of high-purity elements ( $\mathrm{Tb}-3 \mathrm{~N} ; \mathrm{Cu}$ and $\mathrm{Si}-4 \mathrm{~N})$. The synthesis was performed on a water-cooled copper heart in a purified argon atmosphere using $\mathrm{Ti}$ as a getter. The sample was remelted several times to ensure good homogeneity. Subsequently, the product was annealed at $950{ }^{\circ} \mathrm{C}$ for one week.

The quality of the sample was checked by $x$-ray powder diffraction. The diffraction pattern was indexed within the orthorhombic $\mathrm{Gd}_{3} \mathrm{Cu}_{4} \mathrm{Ge}_{4}$-type structure with the lattice parameters $a=13.741(2) \AA, b=6.5513(8) \AA$ and $c=4.1462(5) \AA$, being in agreement with the data reported in [4]. The pattern revealed the existence of a small amount of $\mathrm{Tb}_{2} \mathrm{O}_{3}$ impurity (less than $5 \%$ )

The magnetic measurements were performed in the temperature range $1.7-400 \mathrm{~K}$ and in magnetic fields up to $5 \mathrm{~T}$ using a Quantum Design MPMS-5 SQUID magnetometer. The electrical resistivity was measured over the interval 4.2-300 K employing a standard dc four-probe technique. Current contacts were made using silver paste, and voltage contacts were produced by spark welding. The Seebeck coefficient was measured from 5 to $300 \mathrm{~K}$ by a standard differential method with copper as a reference material. Specific heat studies were carried out by a relaxation method using a Quantum Design PPMS-9 platform. These measurements were made in the temperature range 1.9-300 K under applied magnetic fields up to $9 \mathrm{~T}$.

\section{Results and discussion}

\subsection{Magnetic properties}

The inverse magnetic susceptibility of $\mathrm{Tb}_{3} \mathrm{Cu}_{4} \mathrm{Si}_{4}$ follows the Curie-Weiss law at temperatures above $70 \mathrm{~K}$ (see figure 1). The paramagnetic Curie temperature $\theta_{\mathrm{P}}$ is $19.7(1) \mathrm{K}$ and the effective magnetic moment $\mu_{\mathrm{eff}}$ is 9.51(5) $\mu_{\mathrm{B}}$. The negative sign of $\theta_{\mathrm{P}}$ indicates antiferromagnetic interactions to be dominant. The value of $\mu_{\text {eff }}$ is nearly equal to the theoretical value calculated for a free $\mathrm{Tb}^{3+}$ ion within the Russell-Saunders coupling scheme $\left(9.72 \mu_{\mathrm{B}}\right)$.

At low temperatures the magnetic susceptibility exhibits two anomalies (see figure 2). The first one may be associated with the Néel temperature at about $43 \mathrm{~K}$. From the neutron diffraction data [1] it becomes clear that at this temperature the $2 \mathrm{~d}$ sublattice undergoes magnetic ordering. The other anomaly, occurring at a temperature of $7.5 \mathrm{~K}$, may be attributed to the ordering of the $4 \mathrm{e}$ sublattice, as manifested by the development of a set of magnetic reflections on the neutron diffraction pattern (cf [1]). This interpretation is furthermore supported by the data presented below. In turn, the small anomaly in $\chi(T)$ visible at $2.4 \mathrm{~K}$ probably results from the magnetic ordering of the $\mathrm{Tb}_{2} \mathrm{O}_{3}$ impurity phase.

In figure 3 , the magnetization isotherms measured at several temperatures are shown. No hysteresis loops were observed for any temperature. The curve taken at $1.7 \mathrm{~K}$ shows a 


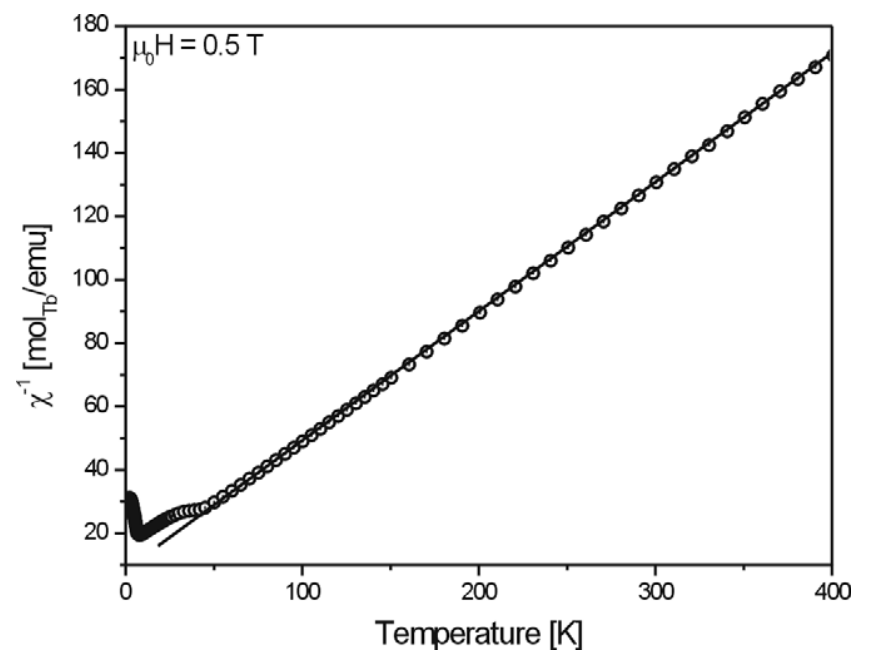

Figure 1. Inverse molar magnetic susceptibility versus temperature for $\mathrm{Tb}_{3} \mathrm{Cu}_{4} \mathrm{Si}_{4}$. The solid line represents the Curie-Weiss fit.

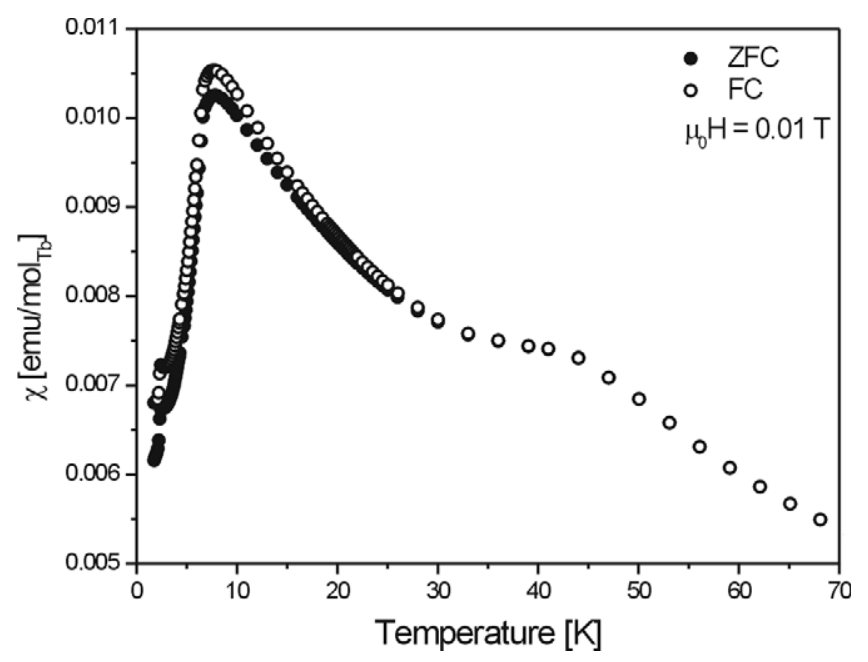

Figure 2. Molar magnetic susceptibility versus temperature for $\mathrm{Tb}_{3} \mathrm{Cu}_{4} \mathrm{Si}_{4}$, measured in a weak magnetic field of $0.01 \mathrm{~T}$ in zero-field-cooled (ZFC) and field-cooled (FC) regimes.

metamagnetic transition at an external field of about $3 \mathrm{~T}$. Such a transition is not visible at any other temperature and therefore one may conclude that the transition involves only the $4 \mathrm{e}$ sublattice. This hypothesis seems to be supported by weaker exchange interactions observed for the $4 \mathrm{e}$ sublattice in comparison to the $2 \mathrm{~d}$ one, as their ordering temperatures differ significantly.

\subsection{Electrical resistivity and thermoelectric power}

The temperature variation of the electrical resistivity of $\mathrm{Tb}_{3} \mathrm{Cu}_{4} \mathrm{Si}_{4}$ is shown in figure 4. At room temperature the resistivity amounts to $44 \mu \Omega \mathrm{cm}$, and on lowering the temperature it decreases in a metallic manner. At low temperatures two distinct kinks are observed at 44 and $7.7 \mathrm{~K}$; however, the corresponding anomalies in the first derivative $\mathrm{d} \rho / \mathrm{d} T$ are visible at 43.2 


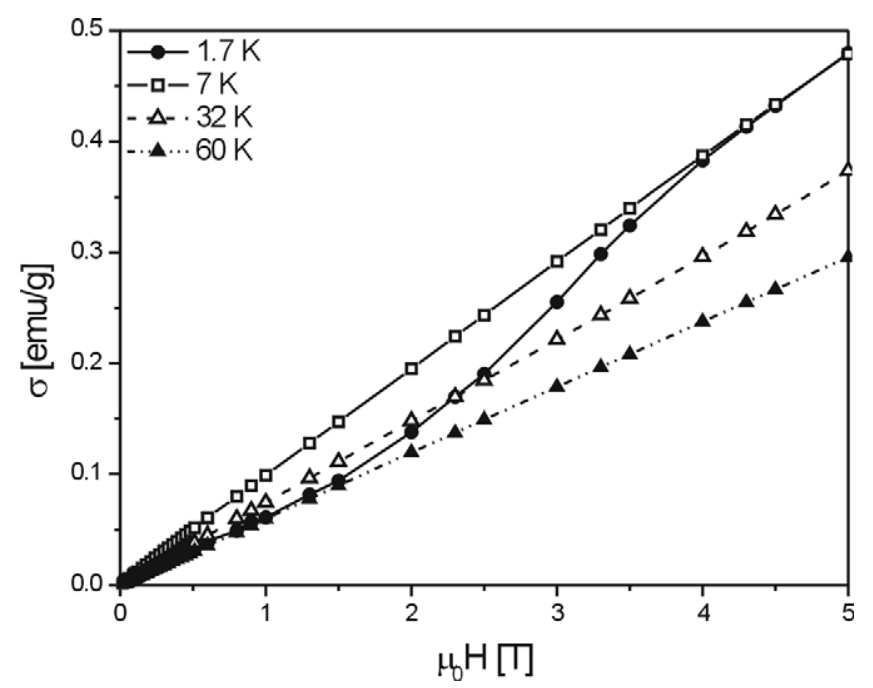

Figure 3. Magnetization versus external magnetic field for $\mathrm{Tb}_{3} \mathrm{Cu}_{4} \mathrm{Si}_{4}$, measured at different temperatures.

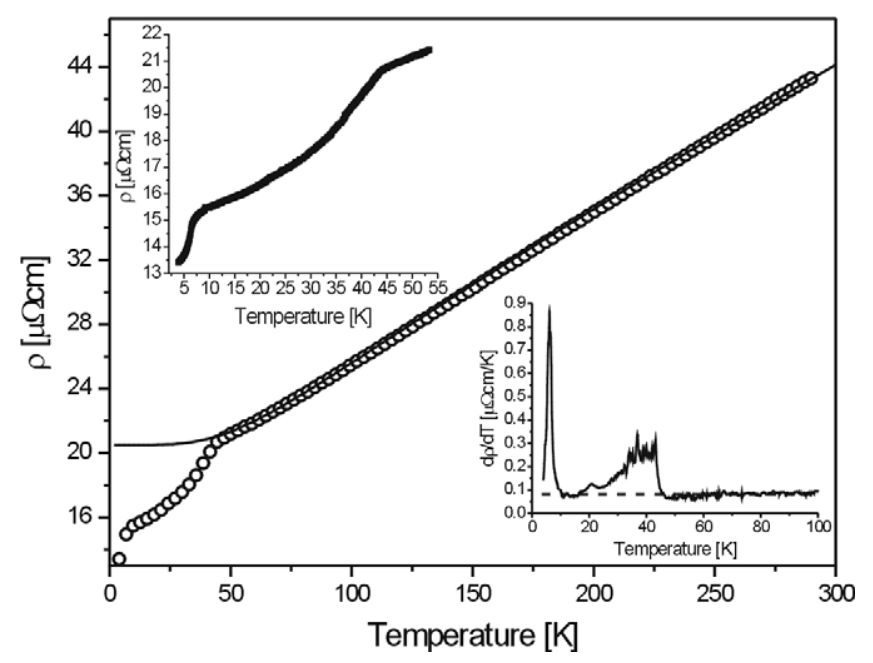

Figure 4. Electrical resistivity versus temperature for $\mathrm{Tb}_{3} \mathrm{Cu}_{4} \mathrm{Si}_{4}$. The solid line represents the Bloch-Grüneisen fit (equation (1)). The insets show the low-temperature region of resistivity and its derivative. In the inset showing the first derivative $\mathrm{d} \rho / \mathrm{d} T$, the dashed line represents the refined value of the asymptotic linear coefficient $R$ from equation (1).

and $6.4 \mathrm{~K}$ respectively (see the insets in figure 4 ). The above-mentioned anomalies can be attributed to the magnetic ordering of the $2 \mathrm{~d}$ and $4 \mathrm{e}$ sublattice, respectively. At about $37 \mathrm{~K} \mathrm{a}$ weak anomaly in $\mathrm{d} \rho / \mathrm{d} T$ curve can be noticed. This anomaly may originate from the first-order transition connected with a change of propagation vector within the $2 \mathrm{~d}$ sublattice.

In the paramagnetic region the electrical resistivity can be approximated by the BlochGrüneisen (BG) formula:

$$
\rho(T)=\left(\rho_{0}+\rho_{\infty}\right)+4 R T\left(\frac{T}{\Theta_{\mathrm{D}}}\right)^{4} \int_{0}^{\frac{\Theta_{\mathrm{D}}}{T}} \frac{x^{5} \mathrm{~d} x}{\left(\mathrm{e}^{x}-1\right)\left(1-\mathrm{e}^{-x}\right)} .
$$




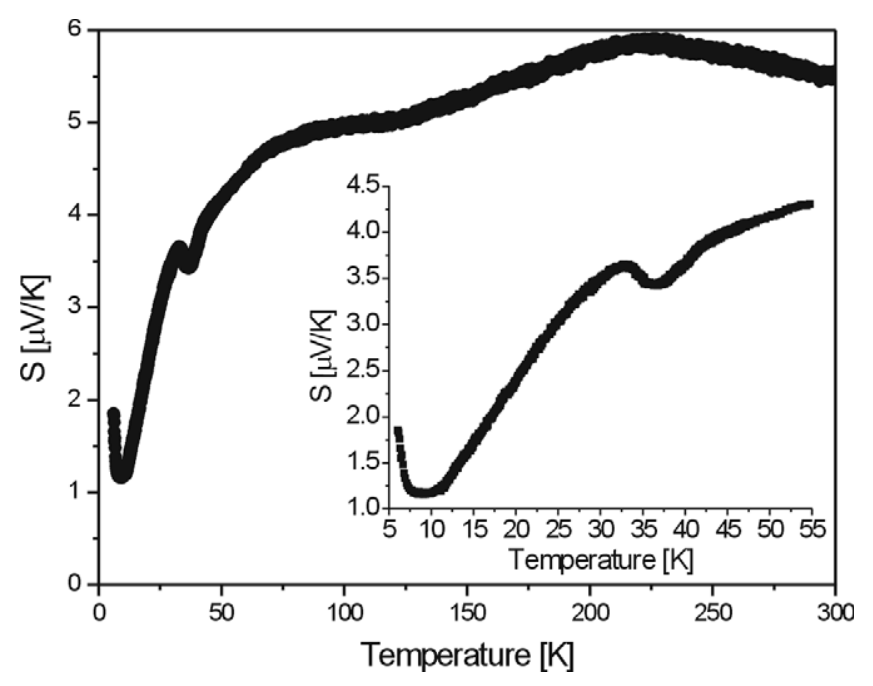

Figure 5. Seebeck coefficient versus temperature for $\mathrm{Tb}_{3} \mathrm{Cu}_{4} \mathrm{Si}_{4}$. Inset: low-temperature region.

The first term in equation (1) describes the scattering of conduction electrons on static defects in the crystal lattice (the residual resistivity $\rho_{0}$ ) and disordered magnetic moments (the spindisorder resistivity $\rho_{\infty}$ ), whereas the second one originates from the electron-phonon scattering $\left(\Theta_{D}\right.$ is the Debye temperature). Fitting the BG model to the experimental data above $55 \mathrm{~K}$ yields the parameters $\rho_{0}+\rho_{\infty}=20.5(1) \mu \Omega \mathrm{cm}, \mathrm{R}=0.0831(7) \mu \Omega \mathrm{cm} \mathrm{K}^{-1}$ and $\Theta_{\mathrm{D}}=311.4(5) \mathrm{K}$. With the residual resistivity $\rho_{0}$ being roughly $13.3 \mu \Omega \mathrm{cm}$, as measured at the lowest temperature, the spin-disorder resistivity $\rho_{\infty}$ is estimated to be close to $7.2 \mu \Omega \mathrm{cm}$. The Debye temperature derived from the resistivity data may be influenced by electron-electron correlations [5]. Equation (1) holds for the temperature region in which the spin-disorder resistivity may be considered as constant, i.e. where any crystal-field effect is negligible. The $\mathrm{d} \rho / \mathrm{d} T$ curve shows that departure from linearity of $\rho(T)$ takes place roughly below $55 \mathrm{~K}$, being indicative of the gradual depopulation of the crystal-field levels.

The temperature behaviour of the thermopower of $\mathrm{Tb}_{3} \mathrm{Cu}_{4} \mathrm{Si}_{4}$ is displayed in figure 5 . The Seebeck coefficient is positive in the entire temperature range studied, thus indicating that the electrical transport is likely dominated by holes [6]. In the paramagnetic region, two broad bumps in $S(T)$ are visible at about 75 and $220 \mathrm{~K}$. The former anomaly may arise due to the so-called phonon drag effect, which usually occurs at a temperature of $0.1-0.3 \Theta_{\mathrm{D}}[7,8]$, while the latter one has no clear origin. Nonlinear behaviour of the thermopower is a characteristic feature of strong electronic correlations in systems with narrow bands in the vicinity of the Fermi energy $[9,10]$. In our case, the $\mathrm{Tb} 4 \mathrm{f}$ level is located deeply below the Fermi level, and thus such an interpretation seems inappropriate.

As shown in the inset to the figure 5, the thermopower exhibits two further anomalies at low temperatures. The minima in $S(T)$ occurring near 37 and $9 \mathrm{~K}$ might be attributed to the magnetic ordering of the two magnetic sublattices, although the characteristic temperatures are slightly different from those derived from the magnetic susceptibility and electrical resistivity data. Better agreement is obtained if the Néel temperature is defined as the inflection point in the $S(T)$ variation above the minimum at $37 \mathrm{~K}$, while the onset of antiferromagnetism in the 4e sublattice is associated with the upturn in $S(T)$ below the minimum at $9 \mathrm{~K}$ (cf the inset to the figure 5). 


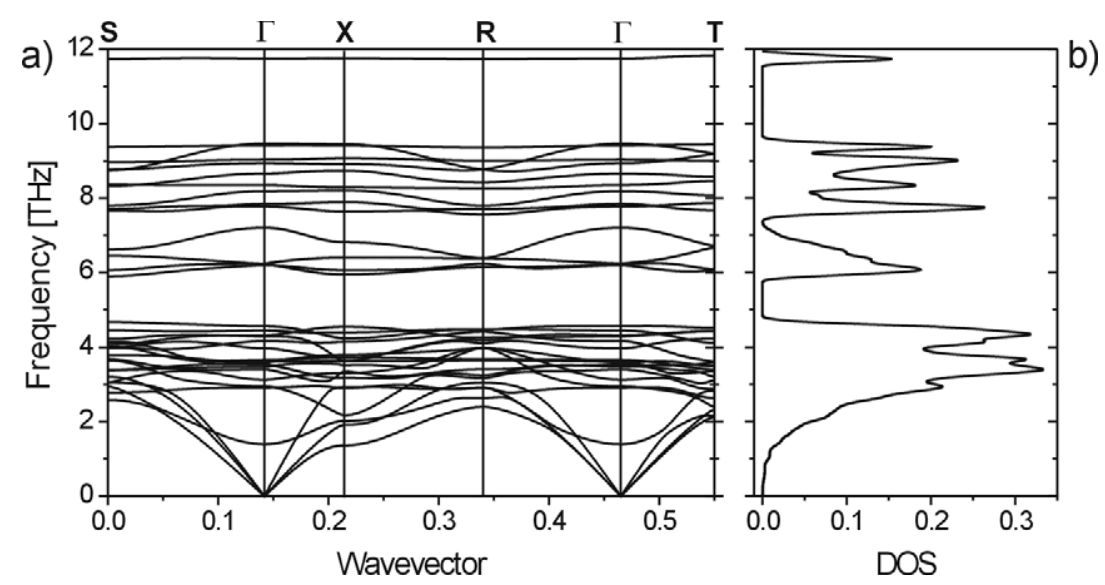

Figure 6. Phonon dispersion relation along the high-symmetry directions within the Brillouin zone (a), and phonon density of states (b). The special points are $S=(1 / 2 ; 0 ; 0), \Gamma=(0 ; 0 ; 0)$, $\mathrm{X}=(-1 / 2 ; 1 / 2 ; 1 / 2), \mathrm{R}=(0 ; 1 / 2 ; 0)$ and $\mathrm{T}=(0 ; 0 ; 1 / 2)$.

\subsection{Specific heat}

3.3.1. Ab initio calculations. In order to provide a deeper discussion of the phononic contribution to the specific heat, density functional theory studies were performed. In the first step, the crystal structure of $\mathrm{Tb}_{3} \mathrm{Cu}_{4} \mathrm{Si}_{4}$ was optimized within the generalized gradient approximation using the VASP program [11]. The calculations were performed for 22 atoms in the $1 \times 1 \times 1$ supercell with periodic boundary conditions. The following valence electron configurations were chosen for the electronic band structure: $\mathrm{Si} 3 \mathrm{~s}^{2} 3 \mathrm{p}^{2}, \mathrm{Cu} 3 \mathrm{~d}^{10} 4 \mathrm{~s}^{1}$, and $\mathrm{Tb} 4 \mathrm{f}^{9}$. The total energy was calculated by summing over the $2 \times 4 \times 4 k$-point grid within reciprocal space. The lattice constants obtained, $a=13.71 \AA, b=6.56 \AA, c=4.17 \AA$, as well as the atomic positions, agree very well with the experimental values.

Afterwards, the phonon frequencies were calculated by means of the direct method implemented in the phonon program [12]. In this method, the Hellmann-Feynman forces are computed by displacing atoms from their equilibrium positions. From the atomic displacements and forces, the force constant elements are obtained by the singular value decomposition method. Having the force constants and dynamical matrices, phonon frequencies can be calculated for each of $k$-points. The phonon density of states (DOS) is derived by the Monte Carlo sampling over the first Brillouin zone. Finally, the lattice heat capacity can be obtained within the harmonic approximation, using a standard thermodynamic approach.

The phonon dispersion curves and DOS are presented in figures 6(a) and (b) respectively. It is worth noting that the optical modes show no degeneracy due to the relatively low symmetry of the lattice considered. On the other hand, there are several soft optical modes that cross the acoustic ones. This is visible in the phonon DOS at low energies, as some Einstein-like spikes are visible, together with Debye-like continuous spectra.

3.3.2. Experimental studies. The temperature variation of the specific heat of $\mathrm{Tb}_{3} \mathrm{Cu}_{4} \mathrm{Si}_{4}$ is shown in figure 7 . At low temperatures two $\lambda$-like peaks at 42 and $6.4 \mathrm{~K}$ are observed, which show full reversibility with respect to heating and cooling (cf the inset to the figure 7 ). Together with the magnetic and electrical data, these two anomalies should be attributed to the magnetic ordering of the $2 \mathrm{~d}$ and $4 \mathrm{e}$ sublattices, respectively. Moreover, an additional feature in $C_{p}(T)$ is visible at $35 \mathrm{~K}$, which has a first-order character (note the clear thermal hysteresis). This 


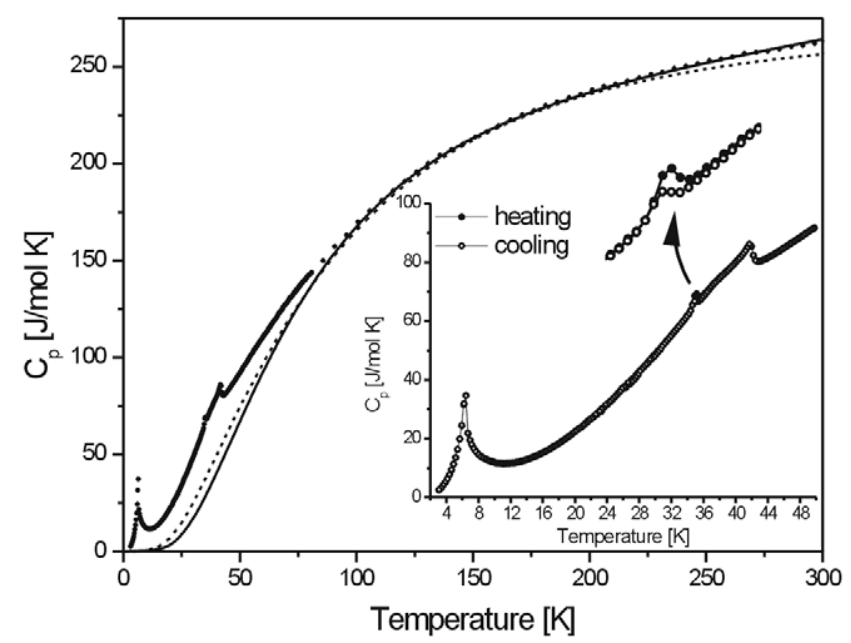

Figure 7. Specific heat versus temperature for $\mathrm{Tb}_{3} \mathrm{Cu}_{4} \mathrm{Si}_{4}$. The solid line represents the fit of nonmagnetic contributions, whereas the dashed one denotes the lattice specific heat calculated using an $a b$ initio method. Inset: low-temperature region. The tiny peak at about $35 \mathrm{~K}$, studied with increasing and decreasing temperature, is magnified.

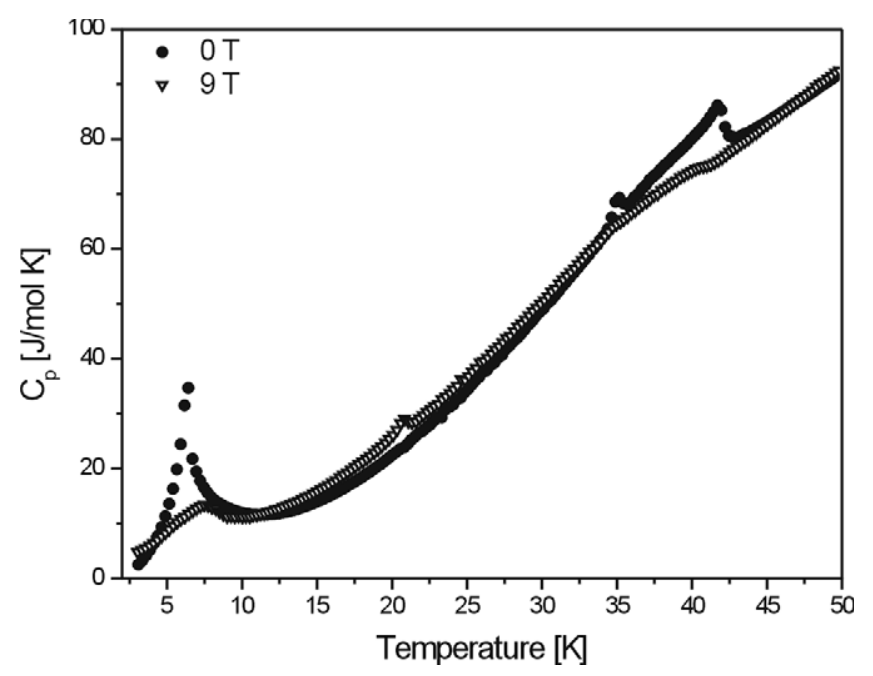

Figure 8. Specific heat versus temperature for $\mathrm{Tb}_{3} \mathrm{Cu}_{4} \mathrm{Si}_{4}$, measured in zero external magnetic field and in $9 \mathrm{~T}$.

latter anomaly may be related to a change of the magnetic structure at the $2 \mathrm{~d}$ sublattice from commensurate into incommensurate one, as previously deduced from the neutron diffraction data [1].

As can be inferred from figure 8, in an external magnetic field of $9 \mathrm{~T}$ the peaks in $C_{p}(T)$ are nearly totally suppressed. At the Néel temperature of about $40 \mathrm{~K}$ only a small residual feature can be noticed, similar to that at the spin-reorientation temperature of $35 \mathrm{~K}$, whereas the transition near $6 \mathrm{~K}$ is entirely obscured by a Schottky anomaly that results from Zeeman splitting of the crystal-field ground state. Most interestingly, upon applying a magnetic 
Table 1. Characteristic temperatures and multiplicities of phonon branches derived from specific heat analysis.

\begin{tabular}{lll}
\hline Part of phonon spectrum & Multiplicity & Characteristic temperature \\
\hline Acoustic (D) & 3 & $\Theta_{\mathrm{D}}=245(3) \mathrm{K}$ \\
& 6 & $\Theta_{E 1}=152(2) \mathrm{K}$ \\
Optical (einstein) & 6 & $\Theta_{E 2}=178(3) \mathrm{K}$ \\
& 6 & $\Theta_{E 3}=221(3) \mathrm{K}$ \\
& 5 & $\Theta_{E 4}=267(4) \mathrm{K}$ \\
& 7 & $\Theta_{E 5}=562(7) \mathrm{K}$ \\
\hline
\end{tabular}

field a small yet clear peak in $C_{p}(T)$ develops at a temperature of $21 \mathrm{~K}$. This feature may manifest a field-induced magnetic or structural phase transition. Complementary neutron and $\mathrm{x}$-ray diffraction experiments in strong magnetic fields are required to address this intriguing hypothesis.

Despite great efforts we have not succeeded in preparing either the Y-based or the La-based nonmagnetic analogue of the $\mathrm{Tb}$ compound studied. The $\mathrm{Y}_{3} \mathrm{Cu}_{4} \mathrm{Si}_{4}$ samples obtained contained as an impurity of about $20 \mathrm{wt} \%$ of $\mathrm{YCu}_{2} \mathrm{Si}_{2}$, while the La-based products showed no trace of the expected Immm crystal structure. To the best of our knowledge, there are no reports in the literature on the formation of $\mathrm{R}_{3} \mathrm{Cu}_{4} \mathrm{Si}_{4}$ compounds with the lightest lanthanides. Therefore, in the analysis of the specific heat measured for $\mathrm{Tb}_{3} \mathrm{Cu}_{4} \mathrm{Si}_{4}$ it was assumed that the phonon and electronic contributions can be approximated by the formula

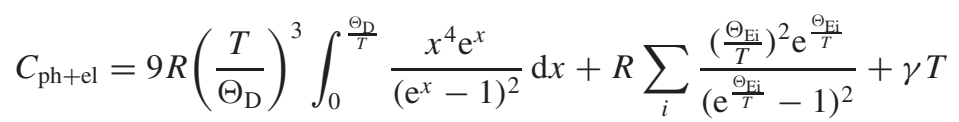

where $\Theta_{D}$ is the Debye temperature, $\Theta_{\mathrm{Ei}}$ are the Einstein temperatures, $\gamma$ is the electronic specific heat coefficient and $R$ is the gas constant. For the compound examined there are three acoustic modes (the first term in equation (2)) and as many as 30 optical modes (the second term in equation (2)). In order to facilitate the analysis, the optical branches were grouped into 'multiple' branches with the multiplicity of 5, 6, 6, 6 and 7. With this simplification the above formula was fitted to the experimental data above $100 \mathrm{~K}$. The least-squares fit is presented in figure 7 by the solid line, and the characteristic temperatures of all phonon branches are listed in table 1. The so-derived Sommerfeld coefficient has a slightly enhanced value of 28(3) $\mathrm{mJ} \mathrm{mol}^{-1} \mathrm{~K}^{-2}$.

According to the $a b$ initio calculations presented in the previous subsection, the predicted lattice specific heat is presented in the figure 7 as well. In general, this curve is similar to lattice contribution obtained by the fitting procedure discussed above (please note that to compare both lattice contributions the electronic part must be excluded from equation (2)). However, within the 125-200 K temperature range this contribution is slightly overestimated compared to the experimental data.

The magnetic specific heat $C_{\text {magn }}$ of $\mathrm{Tb}_{3} \mathrm{Cu}_{4} \mathrm{Si}_{4}$ may be estimated by extracting from the measured $C_{p}(T)$ curve the phonon and electronic contributions. The result is shown in figure 9 . The main contribution of $C_{\text {magn }}$ comes from the Schottky effect given by the standard formula

$$
C_{\text {Schottky }}=\frac{3 R}{T^{2}}\left[\frac{\sum_{i=1}^{13} E_{i}^{2} \mathrm{e}^{-\frac{E_{i}}{T}}}{\sum_{i=1}^{13} \mathrm{e}^{-\frac{E_{i}}{T}}}-\left(\frac{\sum_{i=1}^{13} E_{i} \mathrm{e}^{-\frac{E_{i}}{T}}}{\sum_{i=1}^{13} \mathrm{e}^{-\frac{E_{i}}{T}}}\right)^{2}\right]
$$

in which the parameters $E_{i}$ stand for the energies of the crystal-field (CF) levels (in kelvins). Taking into account the point symmetry of the Tb atoms sites in the unit cell $\left(C_{2 v}\right.$ and $D_{2 h}$ 


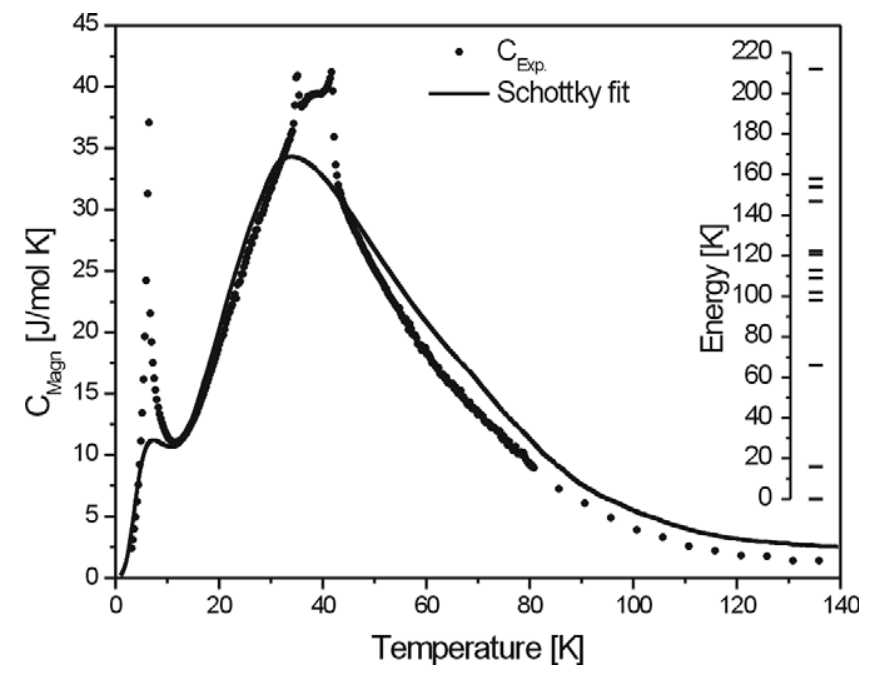

Figure 9. Magnetic specific heat versus temperature for $\mathrm{Tb}_{3} \mathrm{Cu}_{4} \mathrm{Si}_{4}$. The solid curve represents the estimated Schottky contribution. Inset: respective crystal-field splitting scheme.

for the $4 \mathrm{e}$ and $2 \mathrm{~d}$ sites, respectively) a set of nine parameters of the CF Hamiltonian should be considered for each site: $\mathrm{B}_{2}^{0}, \mathrm{~B}_{2}^{2}, \mathrm{~B}_{4}^{0}, \mathrm{~B}_{4}^{2}, \mathrm{~B}_{4}^{4}, \mathrm{~B}_{6}^{0}, \mathrm{~B}_{6}^{2}, \mathrm{~B}_{6}^{4}, \mathrm{~B}_{6}^{6}$. Under such $\mathrm{CF}$ potentials the ${ }^{7} \mathrm{~F}_{6}$ ground multiplets of the non-Kramers $\mathrm{Tb}$ ions split into 13 singlets. The splitting schemes can be different at the two inequivalent Tb sites; however, in order to derive some general conclusions we simplified the analysis by assuming a 'mean' splitting scheme for both of them. The best fit of equation (3) to the experimental data was obtained for the CF scheme presented in the inset to figure 9. Remarkably, the overall CF splitting does not exceed $220 \mathrm{~K}$, and the energy separation of the first excited state from the ground singlet is less than $20 \mathrm{~K}$ $(\sim 1.7 \mathrm{meV})$. Presumably, the exchange energy in the compound studied is larger than this small excitation energy, and therefore it orders magnetically at low temperatures, despite the presence of a nonmagnetic ground state [13].

The magnetic entropy in $\mathrm{Tb}_{3} \mathrm{Cu}_{4} \mathrm{Si}_{4}$, calculated from the formula $S_{\text {Magn }}(T)=$ $\int_{0}^{T} \frac{C_{\mathrm{Magn}}\left(T^{\prime}\right)}{T^{\prime}} \mathrm{d} T^{\prime}$, is presented in figure 10. At room temperature it amounts to $64.5 \mathrm{~J} \mathrm{~mol}^{-1} \mathrm{~K}^{-1}$, thus being very close to the theoretical value of $3 R \ln 13 \approx 65 \mathrm{~J} \mathrm{~mol}^{-1} \mathrm{~K}^{-1}$. This nice conformity is a good measure of the reliability of the performed analysis of the specific heat, which yielded $C_{\text {magn }}(T)$. At the magnetic transition at $6.4 \mathrm{~K}$ the magnetic entropy is about $12.3 \mathrm{~J} \mathrm{~mol}^{-1} \mathrm{~K}^{-1}$, i.e. it is lower than the value of $3 R \ln 2 \approx 17.6 \mathrm{~J} \mathrm{~mol}^{-1} \mathrm{~K}^{-1}$ expected for a doublet ground state, in line with the conclusion derived from the above discussion of the CF effect. At $T_{\mathrm{N}}=42 \mathrm{~K}$ the magnetic entropy is $48.3 \mathrm{~J} \mathrm{~mol}^{-1} \mathrm{~K}^{-1}$, due to the population of higher $\mathrm{CF}$ levels.

\subsection{Total entropy and magnetocaloric effect}

Figure 11(a) displays the total entropy in $\mathrm{Tb}_{3} \mathrm{Cu}_{4} \mathrm{Si}_{4}$ calculated as $S_{\text {Total }}(T)=\int_{0}^{T} \frac{C_{p}\left(T^{\prime}\right)}{T^{\prime}} \mathrm{d} T^{\prime}$ from the experimental data obtained in external magnetic fields of 0,7 and 9 T. Apparently, the most distinct change of the entropy upon applying a magnetic field is associated with the second-order magnetic phase transition at about $6.4 \mathrm{~K}$. From these data the adiabatic temperature change (magnetocaloric effect-MCE) can be calculated, and the results are presented in figure 11(b). The largest adiabatic temperature change is observed at about $11.5 \mathrm{~K}$; 


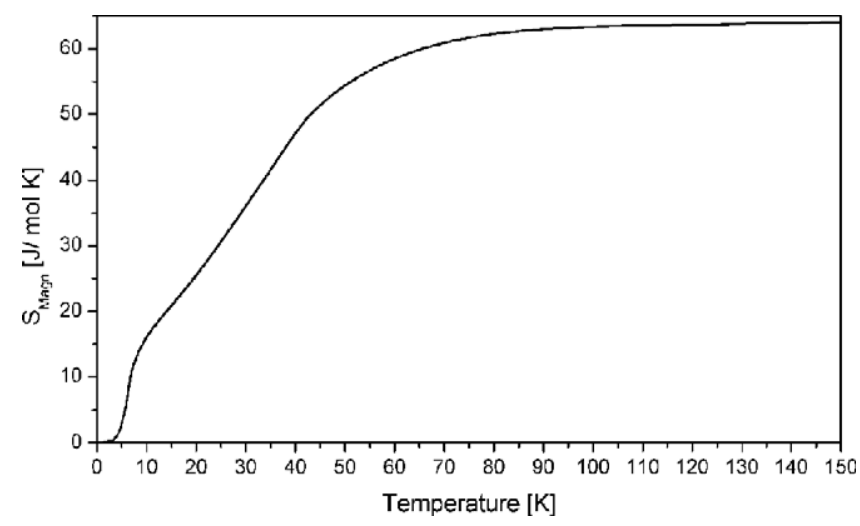

Figure 10. Magnetic entropy versus temperature for $\mathrm{Tb}_{3} \mathrm{Cu}_{4} \mathrm{Si}_{4}$.
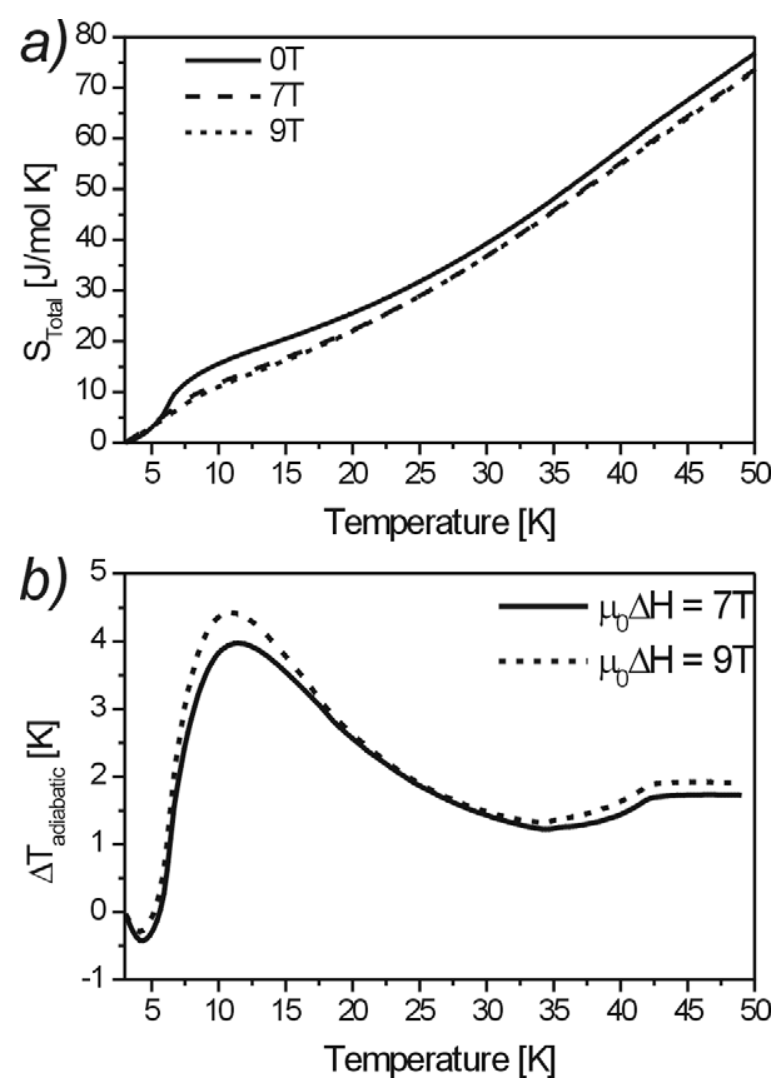

Figure 11. Total entropy (a) and adiabatic temperature change (b) versus temperature for $\mathrm{Tb}_{3} \mathrm{Cu}_{4} \mathrm{Si}_{4}$, derived in different external magnetic fields.

$\Delta T_{\text {adiabatic }}$ reaches $4.5 \mathrm{~K}$ in a field of $9 \mathrm{~T}$. At higher temperatures the positive MCE first gradually diminishes and than slightly rises near the Néel temperature to saturate at a value of about $1.6 \mathrm{~K}$. At low temperatures $\Delta T_{\text {adiabatic }}$ reverses its sign at $5.6 \mathrm{~K}$. This feature is due to the field dependence of the Schottky contribution to the specific heat, which manifests itself as 

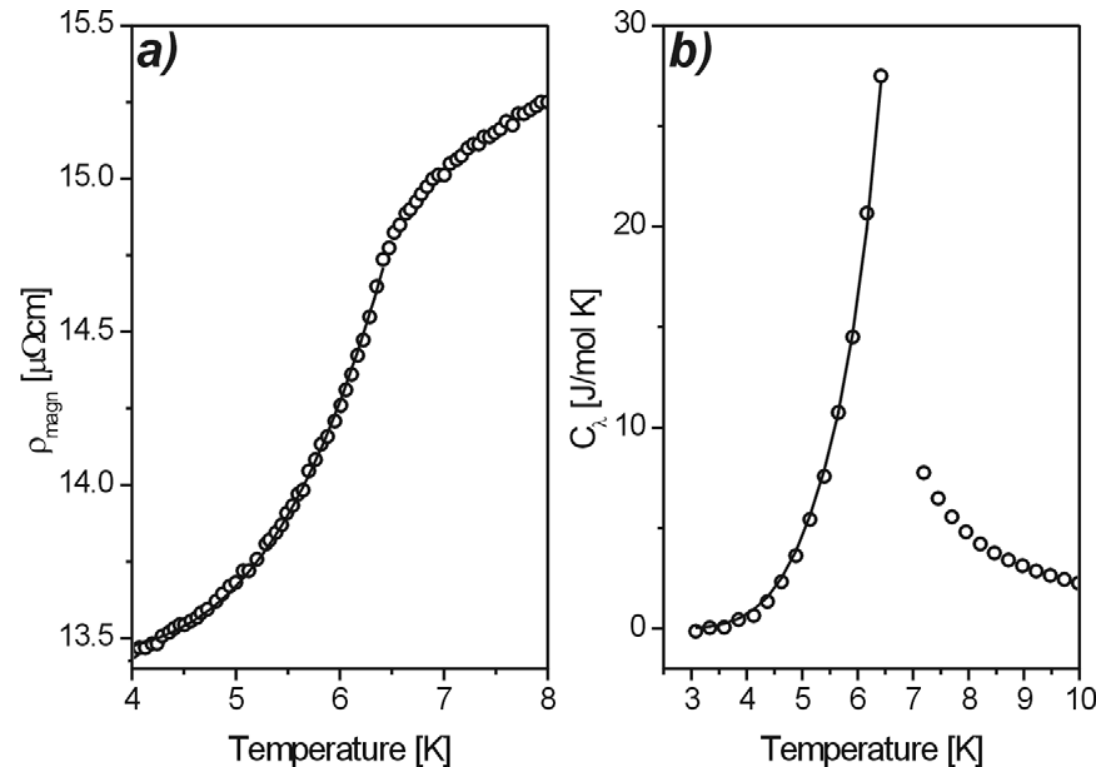

Figure 12. Magnetic contribution to the resistivity (a) and the specific heat (b) versus temperature for $\mathrm{Tb}_{3} \mathrm{Cu}_{4} \mathrm{Si}_{4}$. Solid lines represent the fits according to antiferromagnetic spin-density wave model.

a crossing of the $C_{p}(T)$ variations taken in zero and applied magnetic field, i.e. by shifting the entropy to higher temperatures (cf figure 8).

\subsection{Antiferromagnetic magnons}

In order to discuss the contributions to the electrical and thermal characteristics of $\mathrm{Tb}_{3} \mathrm{Cu}_{4} \mathrm{Si}_{4}$ due to the antiferromagnetic ordering, the temperature dependences $\rho_{\text {magn }}(T)$ and $C_{\lambda}(T)$ were derived by extracting the phonon contribution from the measured electrical resistivity (the second term in equation (1)) and the electron, phonon and Schottky contributions from the measured specific heat (given by equations (2) and (3)). The results are shown in figure 12 . They can be analysed in terms of antiferromagnetic magnons (AFM) theory. The AFM approach predicts for the resistivity the following contribution [14]:

$$
\rho_{\text {magn }}=a \Delta^{2} \sqrt{\frac{T}{\Delta}} \mathrm{e}^{-\frac{\Delta}{T}}\left[1+\frac{3}{2} \frac{T}{\Delta}+\frac{2}{15}\left(\frac{T}{\Delta}\right)^{2}\right]+\rho_{0}
$$

where $\Delta$ is a gap in the AFM spectrum. The parameters derived by a least-squares fit of this formula to the experimental data below $6.5 \mathrm{~K}$ are $a=0.372(7) \mu \Omega \mathrm{cm} \mathrm{K}^{-2}, \rho_{0}=$ 13.340(2) $\mu \Omega \mathrm{cm}$ and $\Delta=33.8(1) \mathrm{K}$. In turn, for the magnetic specific heat the relevant formula for AFM was used [16]:

$$
C_{\lambda}=b T^{-\frac{1}{2}} \mathrm{e}^{-\frac{\Delta}{T}}
$$

and the parameters found for the same temperature range are $b=2.81(1) \times 10^{4} \mathrm{~J} \mathrm{~mol}^{-1} \mathrm{~K}^{-1 / 2}$ and $\Delta=37.1(2) \mathrm{K}$. It is worthwhile noting that the gap values determined from the two different experiments are in a quite good conformity, thus proving the suitability of the analysis performed. 


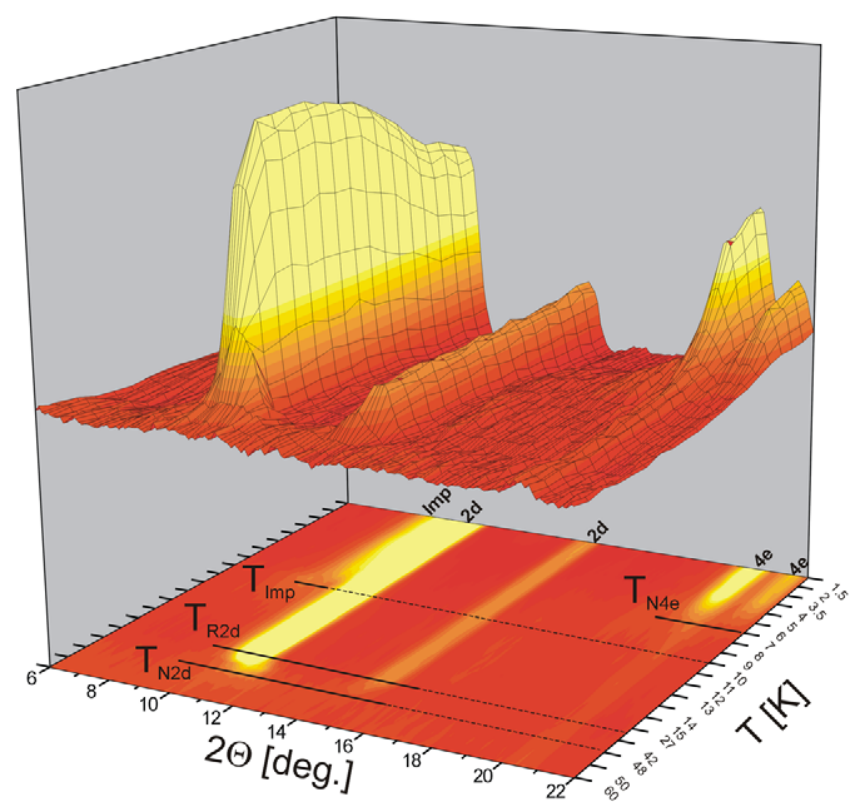

Figure 13. Low-angle neutron diffraction patterns collected at different temperatures for $\mathrm{Tb}_{3} \mathrm{Cu}_{4} \mathrm{Si}_{4}$ (the data taken from [1]). Reflections respective to the $2 \mathrm{~d}$ and $4 \mathrm{e}$ sublattice as well as to the impurity phase are denoted as $2 \mathrm{~d}, 4 \mathrm{e}$, and Imp respectively. $T_{\mathrm{N} 2 \mathrm{~d}}$ and $T_{\mathrm{N} 4 \mathrm{e}}$ are the ordering temperatures for the $2 \mathrm{~d}$ and $4 \mathrm{e}$ sublattice. $T_{\mathrm{R} 2 \mathrm{~d}}$ is the $2 \mathrm{~d}$ sublattice reorientation temperature. $T_{\mathrm{Imp}}$ is the ordering temperature of an impurity (see [1] for details). Note the nonlinear temperature scale.

(This figure is in colour only in the electronic version)

\subsection{Magnetic structure revisited}

Reanalysis of our former neutron diffraction (ND) data [1] brings the following picture of the magnetic structure. At a temperature of $1.5 \mathrm{~K}$ both magnetic sublattices are ordered with different propagations vectors: $(0 ; 1 / 2 ; 0)$ and $(0.231(2) ; 1 / 2 ; 1 / 2)$ for the $2 \mathrm{~d}$ and $4 \mathrm{e}$ sublattice respectively. The magnetic moments in the $2 \mathrm{~d}$ sublattice are equal to $7.9(1) \mu_{\mathrm{B}}$ and they form an angle of $20^{\circ}$ with the $a$-axis; their sequence of signs is $[++]\left(R_{\text {magn }}=16.4 \%\right)$. In the $4 \mathrm{e}$ sublattice the magnitude of magnetic moments is close to 8.1(2) $\mu_{\mathrm{B}}$. The $4 \mathrm{e}$ magnetic moments lie within the $b-c$-plane; they form an angle of $25(4)^{\circ}$ with the $b$-axis, and their sequence of signs is $[++++]\left(R_{\operatorname{magn}}=18.1 \%\right)$. The departure of $2 \mathrm{~d}$ magnetic moments from the $a$ axis direction below $7 \mathrm{~K}$ is reflected in the change of intensity of magnetic reflection at a $2 \Theta$ angle of about $10.5^{\circ}$ (see figure 13). Note that another reflection at a $2 \Theta$ angle of about $15^{\circ}$ originating from the $2 \mathrm{~d}$ sublattice does not change significantly (this is clear evidence of the above-mentioned reorientation of $2 \mathrm{~d}$ magnetic moments). At $7 \mathrm{~K}$ the $2 \mathrm{~d}$ magnetic moments are parallel to the $a$-axis. This reorientation is directly related to disordering of the $4 \mathrm{e}$ sublattice, which takes place at $7 \mathrm{~K}$. In the previously performed ND data analysis, the $4 \mathrm{e}$ sublattice ordering temperature was apparently overestimated. The magnitude of the $4 \mathrm{e} \mathrm{Tb}$ magnetic moment above a temperature of $7 \mathrm{~K}$ was found to be less than 1.6(2) $\mu_{\mathrm{B}}$ [1]. In the light of the new results presented it is clear that above $7 \mathrm{~K}$ only the $2 \mathrm{~d}$ sublattice is ordered. Diffraction peaks at about $20^{\circ}-22^{\circ}(2 \Theta)$ originating from the $4 \mathrm{e}$ magnetic sublattice disappear at $7 \mathrm{~K}$ (see figure 13); moreover, an increase of the background in a diffusive manner can be noticed within this region. This diffusive scattering can be attributed to disordering of the $4 \mathrm{e}$ sublattice. This 
Table 2. Magnetic ordering temperatures of $4 \mathrm{e}$ and $2 \mathrm{~d}$ sublattices, as derived from different experiments.

\begin{tabular}{lll}
\hline Method & $T_{\mathrm{N} 4 \mathrm{e}}(\mathrm{K})$ & $T_{\mathrm{N} 2 \mathrm{~d}}(\mathrm{~K})$ \\
\hline$\chi(T)$ & 7.5 & 43 \\
$\rho(T)$ & 6.3 & 43.2 \\
$S(T)$ & 9 & 37 \\
$C_{p}(T)$ & 6.4 & 42 \\
$\mathrm{ND}$ & 7 & In between 42 and 48 \\
\hline
\end{tabular}

observation is clearly supported by the second-order transition visible as the kink in resistivity as well as the $\lambda$-like anomaly in the specific heat curve, at about $7 \mathrm{~K}$ (see figures 4 and 7 respectively).

In the temperature range between 7 and $50 \mathrm{~K}$ the ND patterns were reanalysed involving $\mathrm{Tb}$ magnetic moments in only the $2 \mathrm{~d}$ sublattice. In this temperature range the orientation of the $2 \mathrm{~d}$ magnetic moments is parallel to the $a$-axis with sequence [++]. The thermal evolution of magnetic ordering of the $2 \mathrm{~d}$ sublattice within this temperature range is as follows.

$7 \mathrm{~K}$-the magnetic moment is equal to $8.2(2) \mu_{\mathrm{B}}$, the propagation vector is $(0 ; 1 / 2 ; 0)$ $R_{\text {magn }}=19.1 \%$;

$10 \mathrm{~K}$-the magnetic moment is equal to $8.2(1) \mu_{\mathrm{B}}$, the propagation vector is $(0 ; 1 / 2 ; 0)$ $R_{\text {magn }}=19.0 \%$;

$13 \mathrm{~K}$-the magnetic moment is equal to $8.1(1) \mu_{\mathrm{B}}$, the propagation vector is $(0 ; 1 / 2 ; 0)$ $R_{\text {magn }}=19.3 \%$;

$15 \mathrm{~K}$-the magnetic moment is equal to $8.0(1) \mu_{\mathrm{B}}$, the propagation vector is $(0 ; 1 / 2 ; 0)$ $R_{\mathrm{magn}}=19.4 \%$;

$42 \mathrm{~K}$-the magnetic moment is equal to $3.4(3) \mu_{\mathrm{B}}$, the propagation vector is $(0 ; 0.515(2) ; 0)$ $R_{\text {magn }}=24.1 \%$;

$48 \mathrm{~K}$-the magnetic reflections are no longer visible, and at about $11^{\circ}(2 \Theta)$ diffusive scattering originating from the disordered $2 \mathrm{~d}$ sublattice is visible.

The change of the $2 \mathrm{~d}$ sublattice propagation vector from a commensurate into an incommensurate one is reflected in the specific heat data as a first-order transition (at about $35 \mathrm{~K}$ - see figure 7). This transition can be easily noticed while the magnetic reflections, originating from the $2 \mathrm{~d}$ sublattice, change position and become broader (see figure 13). In [1] this transition was deduced to occur at about $38 \mathrm{~K}$ according to the AC susceptibility data.

\section{Summary}

The experimental results discussed in this paper clearly show that the two magnetic sublattices in $\mathrm{Tb}_{3} \mathrm{Cu}_{4} \mathrm{Si}_{4}$ order independently at different temperatures. The values of $T_{\mathrm{N}-4 \mathrm{e}}$ and $T_{\mathrm{N}-2 \mathrm{~d}}$ corresponding to the magnetic transitions at the $4 \mathrm{e}$ and $2 \mathrm{~d}$ sites, respectively, and derived via various experimental techniques, are gathered in table 2. Apart from the thermopower data (see section 3.2), all the other experimental results are consistent and they yield critical temperatures of 43(1) and 7.1(6) $\mathrm{K}$ for the $2 \mathrm{~d}$ and $4 \mathrm{e}$ sites, respectively. From the neutron diffraction experiment the ordering temperature of the $2 \mathrm{~d}$ sublattice may not be precisely given; however, it ranges between 42 and $48 \mathrm{~K}$. Both sublattices exhibit antiferromagnetic ordering. Moreover, for the $2 \mathrm{~d}$ sublattice a change of the magnetic structure from incommensurate below the Néel temperature to commensurate below $35 \mathrm{~K}$ is observed in the specific heat data, in agreement with the neutron diffraction results. 
The overall crystal-field splitting in $\mathrm{Tb}_{3} \mathrm{Cu}_{4} \mathrm{Si}_{4}$ does not exceed $220 \mathrm{~K}$. The crystal-field ground states of terbium ions located at both crystallographic sites are singlets. However, their energy separation from the first excited states is less than $2 \mathrm{meV}$, thus making the magnetic ordering possible. The compound exhibits some enhancement of the electronic specific heat $\left(\gamma=28(3) \mathrm{mJ} \mathrm{mol}^{-1} \mathrm{~K}^{-2}\right)$ that may indicate moderate electron-electron correlations. The latter is visible in the complex temperature variation of thermopower as well.

The seemingly independent magnetic behaviour of the rare earth ions occupying two different sublattices and observed inapplicability of the de Gennes scaling for each of the $\mathrm{R}_{3} \mathrm{~T}_{4} \mathrm{X}_{4}$ series suggest that the magnetic orderings in these compounds are governed by exchange interactions that are different from the RKKY ones. It is possible that a proper description of the magnetism in the $\mathrm{R}_{3} \mathrm{~T}_{4} \mathrm{X}_{4}$ intermetallics can be obtained within the Campbell model of local interactions [16].

\section{Acknowledgments}

The authors are grateful to Dr K Gofryk for the thermoelectric power measurement and to Dr E Wawrzyńska for providing the neutron diffraction data.

\section{References}

[1] Wawrzyńska E, Penc B, Stüsser N, Szytuła A and Tomkowicz Z 2003 Solid State Commun. 126527

[2] Wawrzyńska E, Hernandez-Velasco J, Penc B, Sikora W, Szytuła A and Zygmunt A 2003 J. Phys.: Condens. Matter 155279

[3] Wawrzyńska E, Hernandez-Velasco J, Penc B and Szytuła A 2004 J. Magn. Magn. Mater. 280234

[4] Hanel G and Nowotny H 1970 Monatsh. Chem. 101463

[5] Giovannini M, Michor H, Bauer E, Hilscher G, Rogl P and Ferro R 1998 J. Alloys Compounds 28026

[6] Durczewski K and Ausloos M 1994 Phys. Rev. B 4913215

[7] Ziman J M 1967 Electrons and Phonons (Oxford: Oxford University Press)

[8] Blatt F, Schroeder P, Foiles C and Greig D 1976 Thermoelectric Power of Metals (New York: Plenum)

[9] Gottwick U, Gloos K, Horn S, Steglich F and Grewe N 1985 J. Magn. Magn. Mater. 47/48 536

[10] Park J-G and Očko M 1997 J. Phys.: Condens. Matter 94627

[11] Kresse G and Furthmüller J 1996 Comput. Mater. Sci. 615

[12] Parliński K, Li Z Q and Kawazoe Y 1997 Phys. Rev. Lett. 784063 Parliński K 2003 PHONON Software Kraków, Poland

[13] Trammell G T 1963 Phys. Rev. 313625

[14] Fontes M B, Trochez J C, Giordanengo B, Bud'ko S L, Sanchez D R, Baggio-Saitovich E M and Continentino M A 1999 Phys. Rev. B 606781

[15] Akhiezer A I, Baryakhtar V G and Kazanov M I 1960 Usp. Fiz. Nauk 71533

[16] Campbell I A 1972 J. Phys. F: Met. Phys. 2 L47 